\title{
Methanol masers in the Herschel era Putting them in the star formation context
}

\author{
Michele Pestalozzi \\ IAPS - INAF \\ via del Fosso del Cavaliere 100, 00133 Roma, Italy \\ email: michele.pestalozzi@gmail.com
}

\begin{abstract}
Methanol masers are known to be among the most reliable tracers of high-mass stars in early stages of evolution. A number of searches across the Galaxy has yielded to date, a complete census of those masers in two thirds of the Milky Way, providing a catalogue of some 800 sources to be studied in depth. In particular, it is important to characterise the physical properties of the objects hosting methanol masers, and this is possible today using data from the Herschel Space Observatory (HSO). The exceptional spatial resolution of HSO and its wavelength coverage are perfectly tuned to put the methanol maser phase into its star formation context. This paper presents results on the characterisation of methanol maser hosts using Herschel data from the Hi-GAL project, an Open Time Key Project to survey the inner Galactic plane at 5 wavelengths between 70 and $500 \mu \mathrm{m}$.
\end{abstract}

Keywords. Star formation, high-mass stars, masers, methanol

\section{Introduction}

Methanol masers at $6.7 \mathrm{GHz}$ are known to mark, exclusively, high-mass star forming regions in early stages of evolution. After their discovery (Menten 1991) a number of searches have been undertaken in the last two decades, using several strategies: on the one hand, targets were selected on the basis of their Infrared (IR) colours, at that time limited to the wavelength coverage of the IRAS satellite (see e.g. Schutte et al. 1993; van der Walt et al. 1995; Walsh et al. 1998; Szymczak et al. 2000 and several others, a complete list is given in Pestalozzi et al. 2005); on the other hand, large regions of sky have been systematically covered with observations to detect potentially all masers within a certain sesntivity limit (see e.g. Szymczak et al. 2002; Pestalozzi et al. 2002b; Pandian \& Goldsmith 2007; Green et al. 2008). While the first strategy yielded a detection rate of some $15 \%$, the latter technique, although more time consuming, was more efficient in finding new sources with e.g. unexpected IR colours. Examples of this are multiple, some of them are described in Pestalozzi et al. (2002a). The ultimate search for $6.7 \mathrm{GHz}$ methanol masers in the Milky Way is the Methanol MultiBeam (MMB) Survey†, through which two thirds of the Galaxy has been covered, yeilding some 800 sources, $30 \%$ of which are new detections (see e.g. Caswell et al. 2011).

The main idea behind targetted searches was to define the characteristics of the objects that host methanol maser emission, something that, in view of the low detection rates, was only partially achieved. Characterisation of $6.7 \mathrm{GHz}$ methanol maser hosts was also done using Spitzer GLIMPSE and MSX data, as reported by Ellingsen (2006). The latter

$\dagger$ The MMB Survey was conducted using the Parkes Telescope in Australia. The area covered to date comprises the longitude range $60^{\circ}>\ell>-174^{\circ}$. See http://www.jb.man.ac.uk/ research/methanol/ 
study expressed MIR criteria to characterise methanol maser hosts, but apart from a test on a sample of known sources, no searches for new masers were performed on that basis.

The Hi-GAL Survey (High-mass Galactic Plane Survey) $\dagger$ is an Open Time Key Project that has been awarded some 300 hours of observation with the HSO, to survey the inner Galactic Plane in five contiguous bands of the Far Infrared (FIR) dust continuum between 70 and $500 \mu \mathrm{m}$. The unprecedented spatial resolution due to its $3.5 \mathrm{~m}$ mirror, as well as high-dynamical range instruments onboard (in our case two of the three, PACS for wavelengths shorter than $200 \mu \mathrm{m}$ and SPIRE for the wavelength range between 200 and $600 \mu \mathrm{m}$, see Poglitsch et al. 2010; Griffin et al. 2010) make Hi-GAL data the best place to charaterise Galactic cold material (dust) that pinpoints the early stages of star formation. Technical details of the survey are summarised in Molinari et al. (2010).

In this paper we investigate the IR properties of methanol maser hosts that are detected in Hi-GAL. The hypothesis behind this study is that, due to the short duration of the methanol maser phase (between $2.5 \times 10^{4}$ and $4.5 \times 10^{4}$ years, see van der Walt 2005), all methanol maser hosts should basically be coeval. Therefore all differences in their IR appearance are not due to evolution but e.g. as a different level of embededness of the maser pumping object in its primordial dust cocoon. We attempt to disentangle these two effects (evolution and embededness) by studying and characterising of the SEDs of the hosts, allowing, in this way, a precise designation of the methanol maser phase in the context of (high-mass) star formation.

\section{Data: Hi-GAL maps, source extraction, masers, SED fitting}

The data used for the present paper consist of the public MMB catalogue and the latest Hi-GAL maps in all five Herschel bands, as obtained by the pipeline devolped at IAPS-INAF in Rome and described in detail in Traficante et al. (2011). The overlap between available Hi-GAL maps and public positions of methanol masers detected by MMB corresponds to coordinates $20.0^{\circ}>\ell>-71.0^{\circ}$ and $1.0^{\circ}>b>-1.0^{\circ}$. In this portion of the Galactic Plane there are $6356.7 \mathrm{GHz}$ methanol masers. All of them have a Hi-GAL counterpart, albeit not at all Herschel wavelengths.

Compact sources in the Hi-GAL data were extracted around every methanol maser position in a subimage with varying size, depending of the wavelength. In all cases the closest, in angle, on the sky was assigned to the maser as its counterpart. Compact source detection and photometry extraction was performed using CuTEX $\ddagger$, a package developed explicitely to work on images with bright and highly varilable background levels, a characteristic of all Herschel images of regions on the Galactic Plane (see Molinari et al. 2011 for details).

In order to work with an homogeneous set of data, a series of filters were applied to the original source list. For the present work this means only sources for which counterparts at five Herschel wavelengths were found and that were closer than 5 arcseconds to the original maser position. All sources that showed complex SEDs have been excluded; these have to be treated on a sigular basis. The final list contains 421 methanol maser bearing Hi-GAL sources ( $66 \%$ of the starting 635 sources), the ones that are plotted with filled black circles in Fig. 1.

For all sources in the final list, grey body SED fitting was performed, obtaining masses and luminosities for near- and far heliocentric distances. The distribution of several physical properties of methanol maser bearing Hi-GAL sources is shown in Fig. 2.

$\dagger$ See: https://hi-gal.ifsi-roma.inaf.it/higal/

$\ddagger$ CuTEX analyses the second derivative of Herschel images to detect soures and extracts photometric information with Gaussian photometry. 

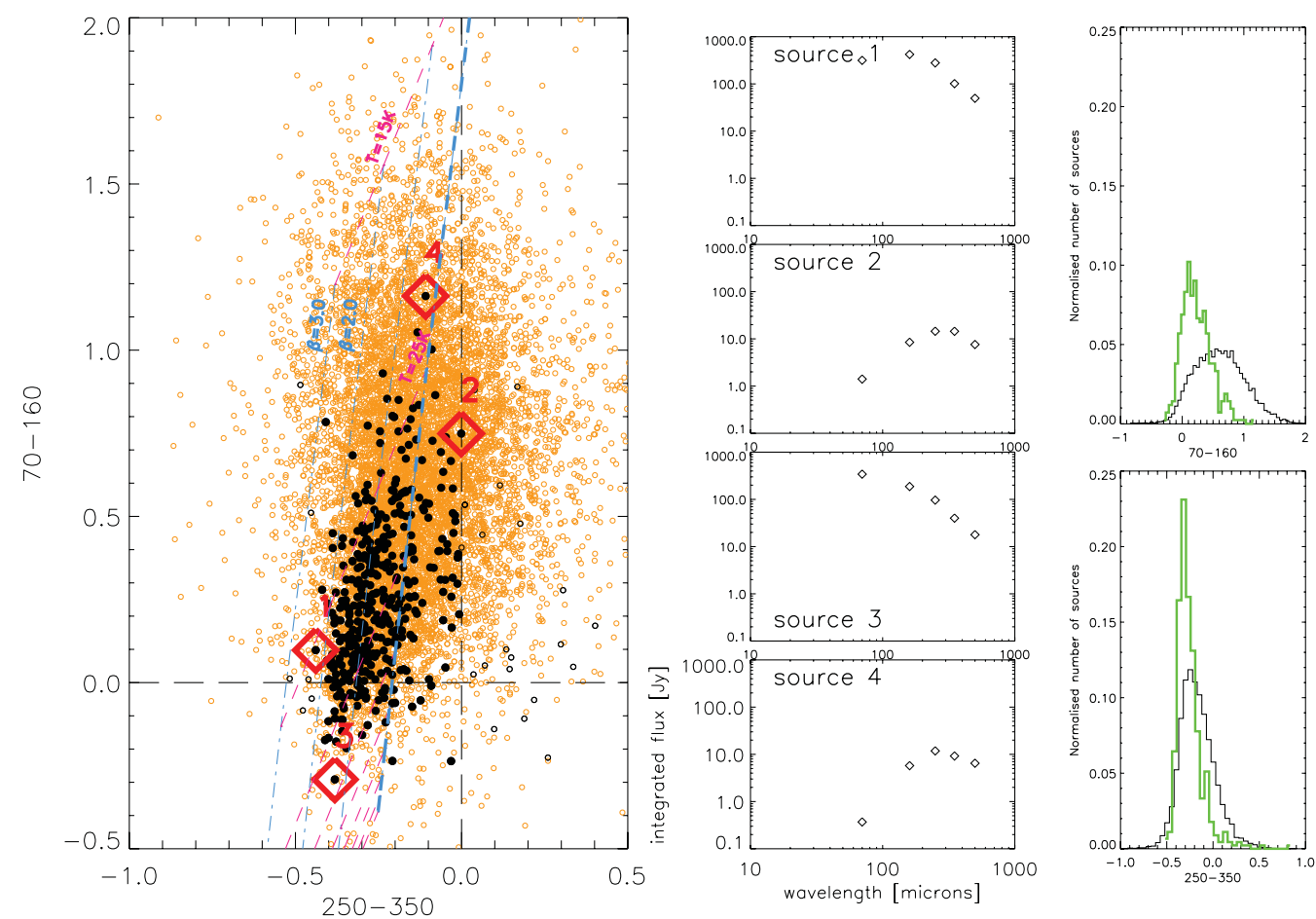

Figure 1. left: Color-color diagram of Hi-GAL sources extracted in the galactic longitude range $20^{\circ}>\ell>-71^{\circ}$ with a clear detection at all 5 Herschel continuum wavelengths. Black symbols indicate the methanol maser bearing sources, orange open circles all other sources. Grey body models are overlaid in blue (equal temperature) and magenta (equal $\beta$ ). Red diamonds indicate the "extreme" sources, for which the SEDs are shown in the four panels in the middle. The two most right panels show the histograms in the colors plotted in the left panel; green lines refer to methanol maser bearing sources, black lines to all other Hi-GAL sources. In both panels histograms have been normalised to the total number of sources.

\section{Results: methanol masers in the context of star formation}

\subsection{Colors}

The left panel of Fig. 1 shows the color-color diagram of all methanol maser bearing sources in the final list (filled black circles), overlaid on all other Hi-GAL sources detected in all 5 Herschel bands. Most of the sources fall in the region with positive 70-160 color and negative 250-350 color, indicating that their SED must peak between 160 and $250 \mu \mathrm{m}$. This is even more true for methanol maser bearing sources, which seem to concentrate in the lower part of that region and mainly to the left of the thick blue line. The latter fact indicates that methanol maser bearing sources are mostly modelled with grey bodies with varying values of $\beta$ (blue lines show grey body models with constant $\beta$ ) and with temperatures between 15 and $25 \mathrm{~K}$ (see magenta lines that show grey bodies with constant temeprature).

The two right-most panels of Fig. 1 show the distribution of sources with and without associated methanol maser (green and black lines, respectively) in the two colors of the left panel. Again, what is immediately clear is the difference in shape between the green and black distribution, the green being more peaked, strongly suggesting that the methanol maser phase is a short one - in the evolution from large dust clump or core to stars. Another important aspect is that methanol maser bearing sources concentrate 

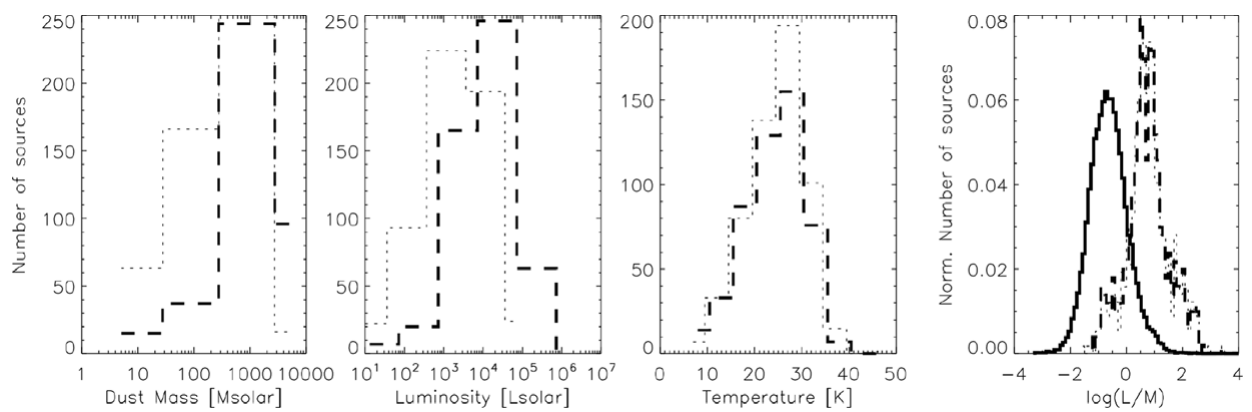

Figure 2. Physical properties of the methanol maser bearing Hi-GAL sources. From left to right: dust mass $\left(M_{\odot}\right)$, bolometric luminosity $\left(L_{\odot}\right)$ and dust temperature $(\mathrm{K})$. Dotted and thick dashed lines refer to quantities for the near- and far heliocentric (kinematic) distances, respectively. The right most panel shows the distribution of the $\mathrm{L} / \mathrm{M}$ ratio for methanol maser bearing sources (dotted and dashed lines) and Hi-GAL sources without methanol maser (solid line), normalised to their total. The peaks of the distribution are distinct, meaning a different overall placement in the L vs M diagram (see Molinari et al. 2008). Also, methanol maser bearing sources seem to have an overall more peaked L/M distribution, suggesting that the methanol maser phase is short.

in the "warmer" part of the diagram, suggesting that methanol masers probably do not appear at the very beginning of the formation of stars, but later on.

Finally, red diamonds indicate the area enclosing Hi-GAL methanol maser bearing sources with simple SEDs. The SEDs of these are displayed in the four panels in the middle of Fig. 1. The quick observation here is that there is a larger spread of colors in the 70-160 than in the 250-350 color.

Note that all temperatures mentioned here refer to what is visible by Herschel, i.e. with a spatial resolution between 5 and 20". At distances of a few kpc, this scale is much larger than the single, maser pumping protostar, explaining the discrepancy in dust temperature between the data presented here and the values obtained in methanol maser pumping models (see e.g. Sobolev et al. 1997). In fact, to allow methanol to evaporate from icy dust grains, a dust temperature of some $100-150 \mathrm{~K}$ is required in the immediate surroundings of the protostar. Only interferometry at millimeter and sub-millimeter wavelengths will allow a connection of the physical properties on the large scales seen by Herschel, with the immediate surroundings of the maser pumping source seen e.g by ALMA.

\subsection{Physical properties and $L / M$ ratio}

Figure 2 shows some physical properties of methanol maser bearing Hi-GAL sources as a result of grey body SED fitting. While mass and luminosity histograms show some difference depending on which distance is adopted (dotted line is near- dashed thick line is far heliocentric distance), temperatures seem to be more stable around $25 \mathrm{~K}$, with a spread between 15 and $35 \mathrm{~K}$. This was also seen in the color-color diagram. As mentioned above, this fact serves to put methanol maser bearing sources into the context of star formation and not to extract the physical properties of the maser pumping source.

A distance independent parameter is the ratio of luminosty to mass, L/M. Comparing the distribution of this ratio among methanol maser bearing sources and all other Hi-GAL sources (Fig. 2, right most panel) one notices that the former peak at values of $\mathrm{L} / \mathrm{M}$ indicating that the luminosity is one order of magnitude higher than the mass, in contrast to all other Hi-GAL sources. In a luminosity versus mass plot, the two distributions can indicate two different evolutionary stages. Models by Molinari et al. (2008) indicate that methanol maser bearing sources lie closer to the end of the accretion phase shortly before, or landing on the ZAMS, while all other Hi-GAL sources are mostly 
concentrated in regions where accretion from the large scale dust envelope is still strongly ongoing. Methanol masers then, seem to appear in an "advanced" stage of evolution, and might not be the very early stages indicator as believed in the past.

A further consideration comes from the shape of the distribution of L/M ratios. General Hi-GAL sources seem to have a broader distribution than methanol maser bearing sources. Circumstantially, this supports what was found in van der Walt (2005) regarding the length of the methanol maser phase. In that paper, methanol masers are calculated to last a few $10^{4}$ years, a much shorter time than the time resolution of SED-based age determination. This would support the idea that methanol maser bearing objects actually host coeval sources. The ultimate statement on this hypothesis will not arrive before extensive observational campaigns with an iterferometer are performed. This will be a challenge for ALMA.

On the basis of all observables presented above, one can conclude that the difference in the IR appearance of several methanol maser bearing sources is probably not an indication of age but rather of different level of embededness of the methanol maser pumping source. This idea is also supported by an example of methanol maser bearing objects in the high-mass star forming region NGC 7538 (see e.g. Pestalozzi et al. (2006)). The three masers in that region are associated with apparently very different sources. Despite this evident difference, these could be hosting very similar objects in their interior, objects that are able to pump a maser.

\section{Acknowledgements}

This work was possible thank to the infatigable work of many people involved in the projects from which data were used here. I would like to mention the MMB team in Manchester and at ATNF Sydney (in particular Gary Fuller, James Caswell and Jimy Green), as well as the Hi-GAL team in Rome (in particular the Hi-GAL P.I. Sergio Molinari as well as Eugenio Schisano and Davide Elia).

\section{References}

Caswell, J. L., Fuller, G. A., Green, J. A., et al. 2011, MNRAS, 1330

Ellingsen, S. P. 2006, ApJ, 638, 241

Green, J. A., Caswell, J. L., Fuller, G. A., et al. 2008, MNRAS, 385, 948

Griffin, M. J., Abergel, A., Abreu, A., et al. 2010, A\&SA, 518, L3

Menten, K. M. 1991, ApJ, 380, L75

Molinari, S., Pezzuto, S., Cesaroni, R., et al. 2008, A\&A A, 481, 345

Molinari, S., Schisano, E., Faustini, F., et al. 2011, A\&SA, 530, A133+

Molinari, S., Swinyard, B., Bally, J., et al. 2010, PASP, 122, 314

Pandian, J. D. \& Goldsmith, P. F. 2007, ApJ, 669, 435

Pestalozzi, M. R., Humphreys, E. M. L., \& Booth, R. S. 2002a, A\&\&A, 384, L15

Pestalozzi, M. R., Minier, V., \& Booth, R. S. 2005, A\&A, 432, 737

Pestalozzi, M. R., Minier, V., Booth, R. S., \& Conway, J. E. 2002b, in Cosmic Masers: from Proto-Stars to Black Holes, IAU Symposium 206, 139

Pestalozzi, M. R., Minier, V., Motte, F., \& Conway, J. E. 2006, A $₫ A$, 448, L57

Poglitsch, A., Waelkens, C., Geis, N., et al. 2010, A\&A, 518, L2

Schutte, A. J., van der Walt, D. J., Gaylard, M. L., \& MacLeod, G. C. 1993, MNRAS, 261, 783

Sobolev, A. M., Cragg, D. M., \& Godfrey, P. D. 1997, MNRAS, 288, L39

Szymczak, M., Hrynek, G., \& Kus, A. J. 2000, A\&AS, 143, 269

Szymczak, M., Kus, A. J., Hrynek, G., Kepa, A., \& Pazdereski, E. 2002, A\& A, 392, 277

Traficante, A., Calzoletti, L., Veneziani, M., et al. 2011, ArXiv e-prints

van der Walt, D. J. 2005, MNRAS, 360, 153

van der Walt, D. J., Gaylard, M. J., \& MacLeod, G. C. 1995, A\&AS, 110, 81

Walsh, A. J., Burton, M. G., Hylard, A. R., \& Robinson, G. 1998, MNRAS, 301, 640 